\title{
Association of TNFSF4 (OX40L) polymorphisms with susceptibility to systemic sclerosis
}

\author{
Pravitt Gourh, ${ }^{1}$ Frank C Arnett, ${ }^{1}$ Filemon K Tan, ${ }^{1}$ Shervin Assassi, ${ }^{1}$ Dipal Divecha, ${ }^{1}$ \\ Gene Paz, ${ }^{1}$ Terry McNearney, ${ }^{2}$ Hilda Draeger, ${ }^{3}$ John D Reveille, ${ }^{1}$ Maureen D Mayes, ${ }^{1}$ \\ Sandeep K Agarwal ${ }^{1}$
}

\begin{abstract}
- Additional data (supplementary table 1) are published online only at http:// ard.bmi.com/content/vol69/ issue3
\end{abstract}

'Division of Rheumatology and Clinical Immunogenetics, Department of Internal Medicine, University of Texas Health Science Center at Houston (UTHSC-H), Houston, Texas, USA

2University of Texas Medical Branch at Galveston, Galveston, Texas, USA

${ }^{3}$ University of Texas Health Science Center at San Antonio, San Antonio, Texas, USA

Correspondence to

Dr Sandeep K Agarwal,

Division of Rheumatology and

Clinical Immunogenetics, 643

Fannin Street, MSB 5.278,

Houston, Texas 77030, USA;

sandeep.k.agarwal@uth.tmc.edu

Accepted 17 August 2009

\section{UNLOCKAD}

This paper is freely available online under the BMJ Journals unlocked scheme, see http:// ard.bmi.com/info/unlocked.dtl

\begin{abstract}
Objective It is increasingly being appreciated that multiple autoimmune diseases share common susceptibility genes. The tumour necrosis factor ligand superfamily member 4 gene (TNFSF4, OX4OL), which encodes for the T cell costimulatory molecule OX40 ligand, has been identified as a susceptibility gene for the development of systemic lupus erythematosus (SLE). Accordingly, the aim of the current study was to investigate the possible association of the TNFSF4 gene region with systemic sclerosis (SSc), an autoimmune disease that leads to the development of cutaneous and visceral fibrosis.
\end{abstract}

Methods A total of 9 single nucleotide polymorphisms (SNPs) in the TNFSF4 gene region, previously associated with susceptibility to SLE, were tested for association with SSc in a collection of 1059 patients with SSc and 698 controls.

Results Case-control comparisons revealed a significant association between susceptibility to SSc and the minor alleles at SNPs rs1234314 (OR $1.20,95 \% \mathrm{Cl} 1.04$ to $\left.1.4, \mathrm{p}_{\mathrm{FDR}}=0.019\right)$, rs 2205960 (OR 1.24, 95\% Cl 1.10 to $1.50, \mathrm{p}_{\mathrm{FDR}}=0.019$ ) and rs844648 (OR 1.16, 95\% Cl 1.01 to $1.30, \mathrm{p}_{\mathrm{FDR}}=0.032$ ) The minor allele at rs 844644 was protective $(0 R$ $0.84,95 \% \mathrm{Cl} 0.70$ to $0.97, \mathrm{p}_{\mathrm{FDR}}=0.038$ ). Analysis of subsets of patients with SSc demonstrated significant associations of the TNFSF4 SNPs with limited and diffuse SSc as well as specific SNPs that were associated with SSc-associated autoantibodies. Finally, the analyses suggest a potential interaction between two TNFSF4 SNPs, rs2205960 and rs844648, with regards to SSc susceptibility.

Conclusions Polymorphisms in the TNFSF4 gene region are associated with susceptibility to SSc and its clinical and autoantibody subsets. TNFSF4 may be another gene that confers risk to multiple autoimmune diseases.

\section{INTRODUCTION}

Recently, a number of reports have identified genes that are associated with the development of multiple autoimmune diseases suggesting that autoimmune diseases share genetic risk factors. ${ }^{1-3}$ For example, PTPN22 has been associated with the development of type I diabetes mellitus (TIDM), rheumatoid arthritis (RA) and systemic lupus erythematosus (SLE). ${ }^{4-6}$ STAT4 is a susceptibility gene for the development of SLE and RA. 8 Together these studies implicate dysregulation of common immune pathways due to polymorphisms in several genes in the development of a variety of autoimmune diseases.
The tumour necrosis factor ligand superfamily member 4 gene (TNFSF4, OX4OL), which encodes for the costimulatory molecule OX40 ligand, has been identified as a susceptibility gene for SLE in multiple studies. ${ }^{9-11}$ To date, polymorphisms in TNFSF4 also have been associated with atherosclerosis, ${ }^{12-14}$ but there are no published reports of the association of TNFSF 4 with autoimmune diseases such as RA, TIDM, or systemic sclerosis (scleroderma, SSc).

SSc is a chronic multisystem disease clinically characterised by progressive fibrosis in the skin and internal organs. ${ }^{15}$ Pathologically, SSc exhibits three cardinal features: inflammation and autoimmunity, vasculopathy and excessive extracellular matrix deposition. ${ }^{15}$ The immune alterations that lead to the development of SSc are unknown, but multiple lines of evidence suggest that SSc may share common immune alterations with SLE. For example, similar to SLE, patients with SSc have a myriad of circulating antinuclear antibodies. ${ }^{16}$ Furthermore, peripheral blood cells from a subset of patients with SLE and SSc have a pattern of upregulation of type I interferon associated gene transcripts. ${ }^{17-19}$ These data now have been extended at the genetic level where SLE and SSc have been associated with polymorphisms in the interferon regulatory factor 5 (IRF5) gene, STAT4, PTPN22 and FAS. . $^{6-80-25}$

Given the association of polymorphisms in the TNFSF 4 gene region with susceptibility to SLE as well as the potential common alterations in immune function and related genes between SLE and SSc, the current study sought to determine if polymorphisms in the TNFSF4 gene region are associated with SSc susceptibility in a large case-control study of North American Caucasian patients with SSc and healthy controls.

\section{METHODS}

\section{Patients with SSc and controls}

A total of 1059 Caucasian patients with SSc and 698 healthy Caucasian controls from the Scleroderma Family Registry and DNA Repository ${ }^{26}$ and the University of Texas Rheumatology Division, ${ }^{27}$ dating from 1986 to present, including the Genetics versus Environment in Scleroderma Outcomes Study (GENISOS) ${ }^{16}$ formed the current cohort. All patients with SSc fulfilled American College of Rheumatology (ACR) preliminary criteria for disease classification ${ }^{28}$ or had at least three of the five CREST (for 'Calcinosis, Raynaud's phenomenon, Eesophageal dysfunction, Sclerodactyly and 
Telangiectasias') features. ${ }^{29} 30$ Patients were not excluded if they had symptoms of myositis or Sjogren's syndrome. Patients in the registry were excluded if they met ACR criteria for SLE. In the division samples, one patient was subsequently diagnosed as having SLE. The patients were classified as limited or diffuse cutaneous SSc according to published criteria. ${ }^{31}$ All participants provided written informed consent and the study was approved by the Committee for the Protection of Human Subjects of The University of Texas Health Science Centre at Houston.

\section{Autoantibody analysis}

Testing for antinuclear antibodies was performed using indirect immunofluorescence (IIF) and HEp-2 cells as antigen substrate (Antibodies Inc, Davis, California, USA). Anti-centromere antibodies (ACA) were determined by their distinctive IIF pattern on HEp-2 cells. ${ }^{32}$ Autoantibodies to topoisomerase I (ATA) were determined by passive immunodiffusion (Inova Diagnostics, San Diego, California, USA). Anti-RNA polymerase III antibodies (ARA) were determined by enzyme-linked immunoassay (MBL, Nagoya, Japan).

\section{SNP selection and genotyping}

Single nucleotide polymorphisms (SNPs) in the TNFSF4 gene region were selected based on TNFSF 4 variants identified in two independent candidate gene studies in SLE. 911 Genomic DNA was extracted from peripheral blood with the PureGene genomic DNA isolation kit (Gentra Systems, Minneapolis, Minnesota, USA). The following TNFSF4 SNPs were genotyped: rs1234314, rs1234315, rs2205960, rs844644, rs12039904, rs2795288, rs844648, rs844665 and rs10912580, using an ABI TaqMan SNP genotyping assay from Applied Biosystems (Foster City, California, USA). ABI 7900HT real-time thermocycler was used to perform the plate reading. Automated allele calling was performed by allelic discrimination plots using SDS V.2.3 software (Applied Biosystems). Multiple positive (Centre d'Etude du Polymorphisme Humain (CEPH) samples) and negative controls were used in each genotyping assay and allele calls were verified with HapMap data for validation.

\section{Statistical analysis}

Statistical analyses were performed using SAS V.9.1.3 (SAS Institute Inc, Cary, North Carolina, USA) software. HardyWeinberg equilibrium (HWE) testing was performed using the exact test for HWE. Allelic and genotypic associations were calculated using the standard Pearson $\chi^{2}$ test or Fisher exact test. Statistical significance was considered at $p<0.05$ after correcting for multiple comparisons using a false discovery rate method $\left(p_{\mathrm{FDR}}\right){ }^{33}$ Logistic regression analysis was performed to confirm these associations. Measures of pairwise linkage disequilibrium (LD) were determined using Haploview (Whitehead Institute for Biomedical Research, Cambridge, Massachusetts, USA). SAS was used for overall ('omnibus test') haplotype frequency tests as well as for haplotype-specific tests. The 'omnibus test' was evaluated to detect differences in overall haplotype frequency profiles between SSc and controls. Classification and regression tree (CART) analysis was performed to explore interactions between SNPs (CART V.6.0; Salford Systems, San Diego, California, USA) using gender and SNPs of TNFSF4 as nominal categorical variables to predict the outcome of SSc. ${ }^{34}$ Logistic regression analysis was performed to verify the SNP interaction observed in CART analysis.

\section{RESULTS}

\section{Linkage disequilibrium in the TNFSF4 gene region in healthy controls}

A candidate gene association study was conducted using 1059 Caucasian patients with SSc and 698 healthy controls. Clinical and serological data for the patients with SSc are presented in table 1. Nine TNFSF4 SNPs previously associated with susceptibility to SLE were genotyped in patients with SSc and controls. All SNPs studied in the TNFSF4 gene region were in HWE in Caucasian controls (see supplementary material). Pairwise LD was calculated by $\mathrm{D}^{\prime}$ and $\mathrm{r}^{2}$ for TNFSF4 SNPs typed in Caucasian controls (figure 1).

Table 1 Clinical and serological characteristics of patients with systemic sclerosis

\begin{tabular}{ll}
\hline Characteristic & Value \\
\hline Controls: & \\
Gender & $(\mathrm{N}=698)^{*}$ \\
$\quad$ Female & $334(50.8)$ \\
$\quad$ Male & $323(49.2)$ \\
Systemic sclerosis: & \\
Gender & $(\mathrm{N}=1059)$ \\
$\quad$ Female & $935(88.3)$ \\
$\quad$ Male & $124(11.7)$ \\
Skin involvement & $(\mathrm{N}=1004)$ \\
$\quad$ Limited systemic sclerosis & $611(60.9)$ \\
$\quad$ Diffuse systemic sclerosis & $393(39.1)$ \\
SSc-associated autoantibodies & $(\mathrm{N}=1059)$ \\
$\quad$ Anti-centromere & $300(28.3)$ \\
Anti-topoisomerase I & $174(16.4)$ \\
Anti-RNA polymerase III & $193(18.2)$ \\
$\quad$ Neither anti-centromere, anti-topoisomerase I, & $392(37.1)$ \\
$\quad$ or anti-RNA polymerase III & \\
\hline
\end{tabular}

Values are number (\%).

* Missing gender information on 41 controls.

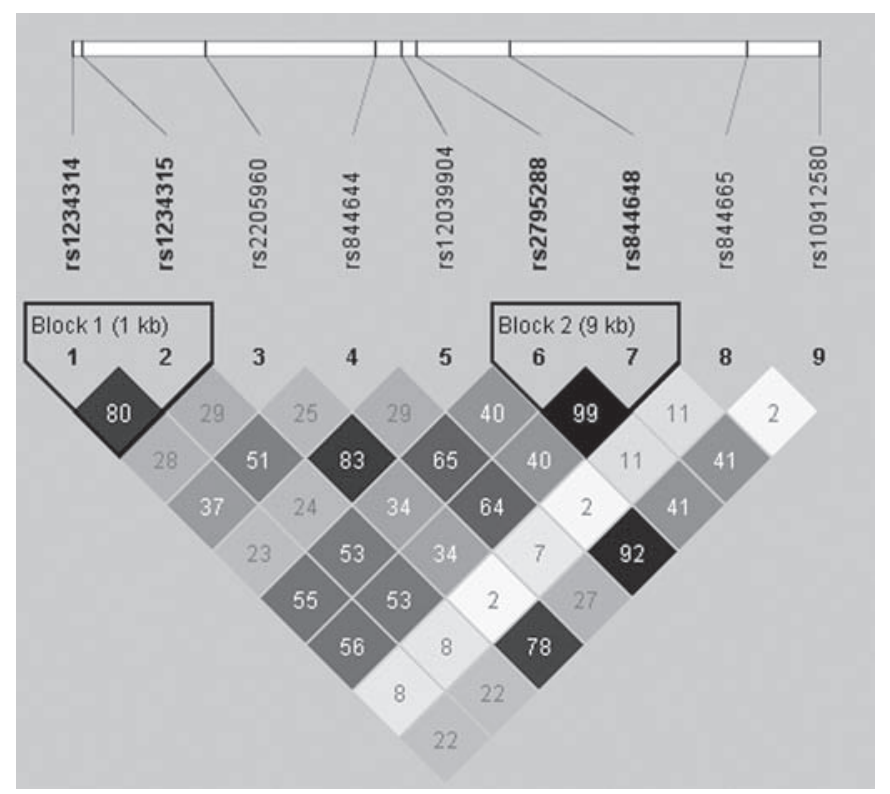

Figure 1 Linkage disequilibrium (LD) and haplotype block structure of the tumour necrosis factor ligand superfamily member 4 gene (TNFSF4) within healthy controls. Blocks connecting pairs of single nucleotide polymorphisms (SNPs) are shaded according to the strength of the linkage disequilibrium between the SNPs, from 0.0 (white) to 1.0 (black), as measured by the disequilibrium coefficient $D^{\prime}$. As an additional measure of strength of $L D, r^{2}$ values are given as numerical values within each box. 
The TNFSF4 SNPs rs1234314-rs1234315, rs2205960-rs12039904, rs2205960-rs10912580, rs12039904-rs10912580 and rs2795288rs844648 showed LD in the Caucasian population $\left(r^{2} \geq 0.80\right)$. Given the tight LD of these SNPs, rs1234315, rs12039904, rs10912580 and rs2795288 were not used in subsequent analyses and the case-control comparisons were performed on the remaining five SNPs tagging the TNFSF4 region.

\section{TNFSF4 polymorphisms and association with SSc}

Case-control comparisons for genotypes using the $\chi^{2}$ test demonstrated significant association for multiple SNPs in the TNFSF 4 gene region (table 2). These associations remained significant even after controlling for multiple comparisons using the false discovery rate method. Compared to controls, patients with SSc had a higher frequency of the minor allele at rs1234314 (OR 1.2, $95 \%$ CI 1.04 to 1.4 ), rs2205960 (OR 1.2, 95\% CI 1.1 to 1.5 ) and rs844648 (OR 1.2, 95\% CI 1.01 to 1.3). The minor allele frequency at rs844648 was less common in patients with SSc compared to controls (OR $0.8,95 \%$ CI 0.7 to 0.97 ). These data were confirmed using logistic regression analyses after controlling for gender (figure 2). No significant difference was observed between patients with SSc and controls for the rs844665 variant.

Table 2 Distribution of the tumour necrosis factor ligand superfamily member 4 gene (TNFSF4) SNP genotype in North American Caucasian patients with SSc and normal controls*

\begin{tabular}{|c|c|c|c|c|c|c|}
\hline \multirow[b]{2}{*}{$\begin{array}{c}\text { SNP } \\
\text { (minor allele) }\end{array}$} & \multirow[b]{2}{*}{ Position (bp) } & \multicolumn{2}{|c|}{ MAF (\%) } & \multicolumn{3}{|c|}{ Allelic $\chi^{2} p$ value and $\mathrm{OR}$} \\
\hline & & $\begin{array}{r}\text { Controls } \\
(\mathrm{N}=698)\end{array}$ & $\begin{array}{c}\text { SSc } \\
(\mathrm{N}=1059)\end{array}$ & $\mathbf{p}_{u}$ & $\mathbf{p}_{\mathrm{FDR}}$ & OR (95\% CI) \\
\hline rs1234314 (G) & 171444015 & 41.3 & 45.8 & 0.01 & 0.019 & 1.20 (1.04 to 1.4$)$ \\
\hline rs2205960 (T) & 171458098 & 21 & 25.0 & 0.01 & 0.019 & 1.24 (1.1 to 1.5$)$ \\
\hline rs844644 (A) & 171476118 & 49.1 & 44.7 & 0.01 & 0.019 & $0.84(0.7$ to 0.97$)$ \\
\hline rs844648 (A) & 171490486 & 41.4 & 45.2 & 0.03 & 0.038 & 1.16 (1.01 to 1.3$)$ \\
\hline rs844665 (T) & 171515580 & 7.3 & 8.3 & 0.31 & 0.31 & $1.19(0.9$ to 1.5$)$ \\
\hline
\end{tabular}

${ }^{*}$ Controls are used as reference for all comparisons.

$\mathrm{bp}$, base pairs; MAF, minor allele frequency; $\mathrm{p}_{\mathrm{FDR}}$, corrected $\mathrm{p}$ values using Benjamini and Hochberg false discovery rate method; $p_{u}$ uncorrected $\chi^{2} p$ values; $S S c$, systemic sclerosis; SNP, single nucleotide polymorphism.
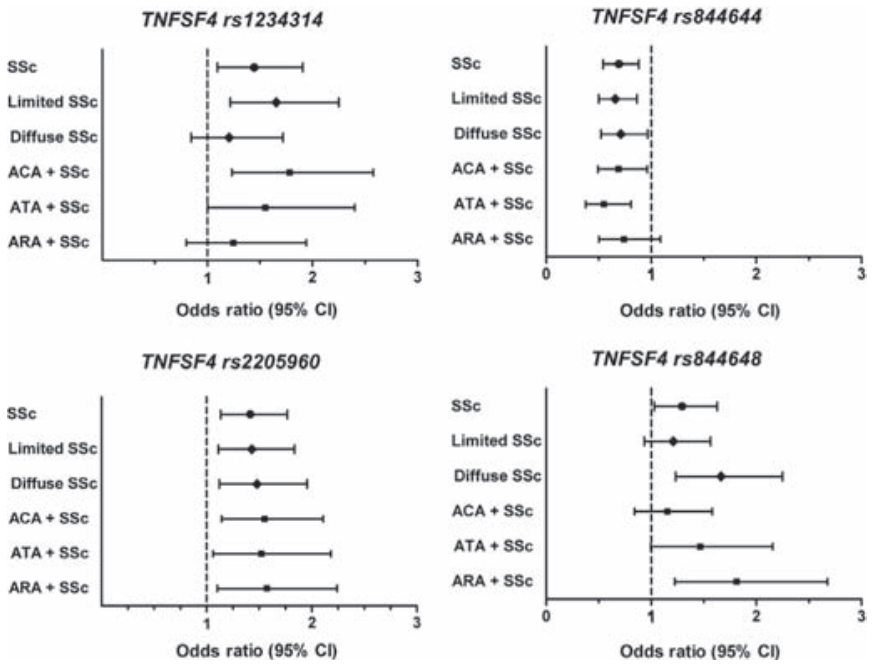

Figure 2 Estimated risk of the tumour necrosis factor ligand superfamily member 4 gene (TNFSF4) single nucleotide polymorphisms (SNPs) in systemic sclerosis (SSc) and subsets of SSc compared to controls by logistic regression controlling for gender.
Analysis of the 5-SNP marker set (rs1234314, rs2205960, rs844644, rs844648, rs844665) in the TNFSF4 revealed 5 out of 28 specific 5 -marker haplotypes that were associated with $\mathrm{SSc}$ (table 3). Four risk haplotypes were identified: C-G-A-A-C $\left(p<10^{-4}\right)$, G-G-C-G-C ( $\left.=0.0006\right)$, G-G-C-G-T $\left(p<10^{-4}\right)$ and G-T-C-G-C $\left(p<10^{-4}\right)$. Consistent with the individual SNP $3 \times 2$ $\chi^{2}$ analysis, the C-G-A-G-C was found to be protective for the development of SSc $\left(p<10^{-4}\right)$. These data support the genetic associations of SNPs in TNFSF 4 with SSc susceptibility observed in the case-control comparisons in table 2.

\section{TNFSF4 polymorphisms and association with subsets of SSc}

$\mathrm{SSc}$ is a clinically heterogeneous disease that is divided into two main clinical subsets based on the extent of cutaneous involvement: limited (1SSc) and diffuse (dSSc). ${ }^{31}$ Furthermore, the presence of SSc-associated autoantibodies such as ACA, ATA and ARA is associated with distinct clinical subsets characterised by extent of cutaneous involvement and pattern of organ involvement. ${ }^{35}$ Several studies have recently shown that certain genetic polymorphisms may have stronger associations with particular phenotypic subsets. ${ }^{21} 36$ Therefore, we compared the frequency of TNFSF 4 SNPs within the clinical and autoantibody subsets of patients with SSc (table 4).

The minor alleles at TNFSF4 SNPs rs1234314 and rs2205960 were more common in 1SSc compared to controls. No differences were observed with rs844644 and rs844648 between 1SSc and controls. Compared to controls, the patients with dSSc had an increased frequency of the minor alleles at TNFSF4 SNPs rs1234314, rs2205960 and rs844648 and decreased frequency of the minor allele at rs844644. Comparison of patients with and without pulmonary fibrosis or pulmonary hypertension did not reveal any differences in the frequency of the SNPs in the TNFSF4 gene region.

Using the SSc-associated autoantibodies to subset patients with SSc, a significant association was observed with rs 1234214 and ACA-positive (OR 1.3, 95\% CI 1.1 to 1.6) and ATA-positive patients with SSc (OR 1.3, 95\% CI 1.02 to 1.7) compared to controls. The minor allele at rs2205960 was more frequent in ATApositive SSc compared to controls (OR 1.4, 95\% CI 1.1 to 1.9). Interestingly, only rs844648 was noted to be associated with ARA-positive SSc compared to controls (OR 1.4, 95\% CI 1.1 to 1.8). No significant differences were noted in the SSc-associated autoantibody subsets of SSc compared to controls at rs844644.

Additional analyses were performed using logistic regression with gender added to the model (figure 2). The strongest associations were observed with rs2205960, where the minor allele was observed with increased frequency in 1SSc and dSSc as well

Table 3 Haplotype analysis of the 5-SNP marker set in the tumour necrosis factor ligand superfamily member 4 gene (TNFSF4) in Caucasian patients with SSc and controls

\begin{tabular}{lccrcc}
\hline Haplotype $^{*}$ & Controls $(\mathbf{N}=\mathbf{6 9 4})$ & $\mathbf{S S c}(\mathbf{N}=\mathbf{1 0 5 0})$ & $\chi^{\mathbf{2}}$ & $\mathbf{p}_{\mathbf{U}}$ & Effect direction \\
\hline OMNIBUS & $\mathrm{NA}$ & $\mathrm{NA}$ & 164.3 & $<0.0001$ & \\
C-G-A-A-C & $0.6 \%$ & $4.9 \%$ & 52.6 & $<0.0001$ & Risk \\
C-G-A-G-C & $43.4 \%$ & $35.1 \%$ & 25.3 & $<0.0001$ & Protective \\
G-G-C-G-C & $0.4 \%$ & $1.7 \%$ & 12.1 & 0.0006 & Risk \\
G-G-C-G-T & $0.0 \%$ & $1.4 \%$ & 20.6 & $<0.0001$ & Risk \\
G-T-C-G-C & $0.6 \%$ & $3.9 \%$ & 37.6 & $<0.0001$ & Risk \\
Others $^{\dagger}$ & $55.0 \%$ & $53.0 \%$ & & & Neutral \\
\hline
\end{tabular}

*The order of the SNPs is rs1234314-rs2205960-rs844644-rs844648-rs844665. 'Includes 23 other haplotypes.

NA, not applicable; $p_{u}$, uncorrected $\chi^{2} p$ values; SSc, systemic sclerosis; SNP, single nucleotide polymorphism. 
Table 4 Distribution of the tumour necrosis factor ligand superfamily member 4 gene (TNFSF4) SNP alleles in North American Caucasian patients with SSc and normal controls*

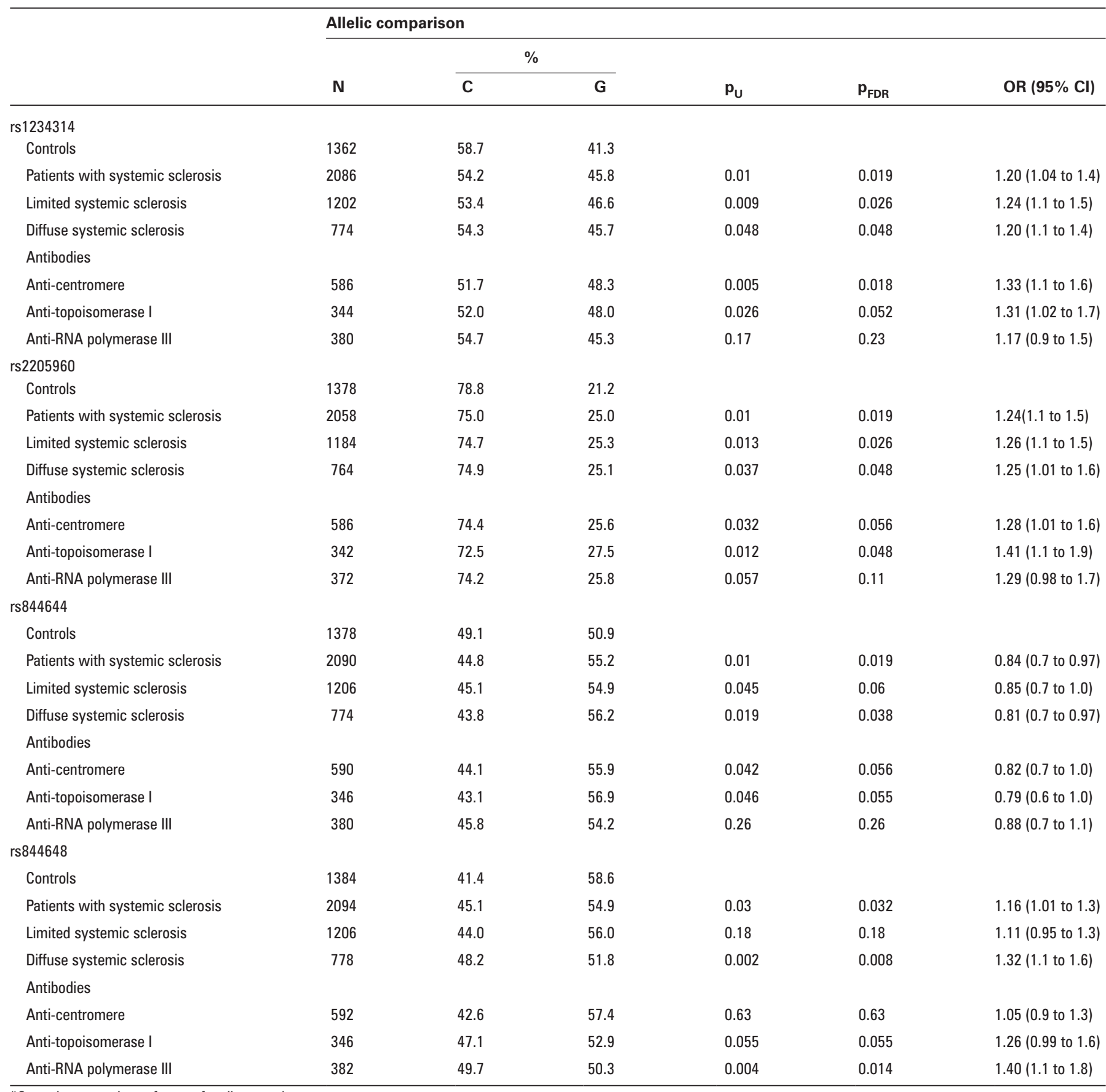

${ }^{*}$ Controls are used as reference for all comparisons.

$\mathrm{p}_{\mathrm{FDR}}$, corrected $\mathrm{p}$ values using Benjamini and Hochberg false discovery rate method; $\mathrm{p}_{\mathrm{U}}$, raw $\chi^{2} \mathrm{p}$ values; SSc, systemic sclerosis; SNP, single nucleotide polymorphism.

as the autoantibody subsets. The minor allele at rs844644 was protective in all groups except for the patients who were ARA positive. With regards to rs1234314, the associations remained in 1SSc, ACA and ATA groups. However, the association of rs1234314 was no longer observed after controlling for gender in the logistic regression analyses. Lastly, with regards to rs844648, logistic regression confirmed the association with $\mathrm{dSSc}$ and ARA. Together these data suggest that different polymorphisms in the TNFSF4 gene region are associated with susceptibility to the distinct autoantibody and skin subsets in SSc.

\section{CART analysis for SNP interaction in the TNFSF4 gene region}

To identify the SNP(s) in TNFSF4 that play a more prominent role in SSc susceptibility, CART analysis was performed. ${ }^{37}$ Accordingly, the five TNFSF4 polymorphisms and gender were entered as variables in the CART model. CART analysis interactively segregates participants into two subgroups using the most powerful variable classifier for SSc.

Figure 3 depicts female gender as the first split. In women, the 'TT/GT' genotype of the TNFSF4 variant (rs2205960) increased risk for SSc as compared to the 'GG' wild-type 


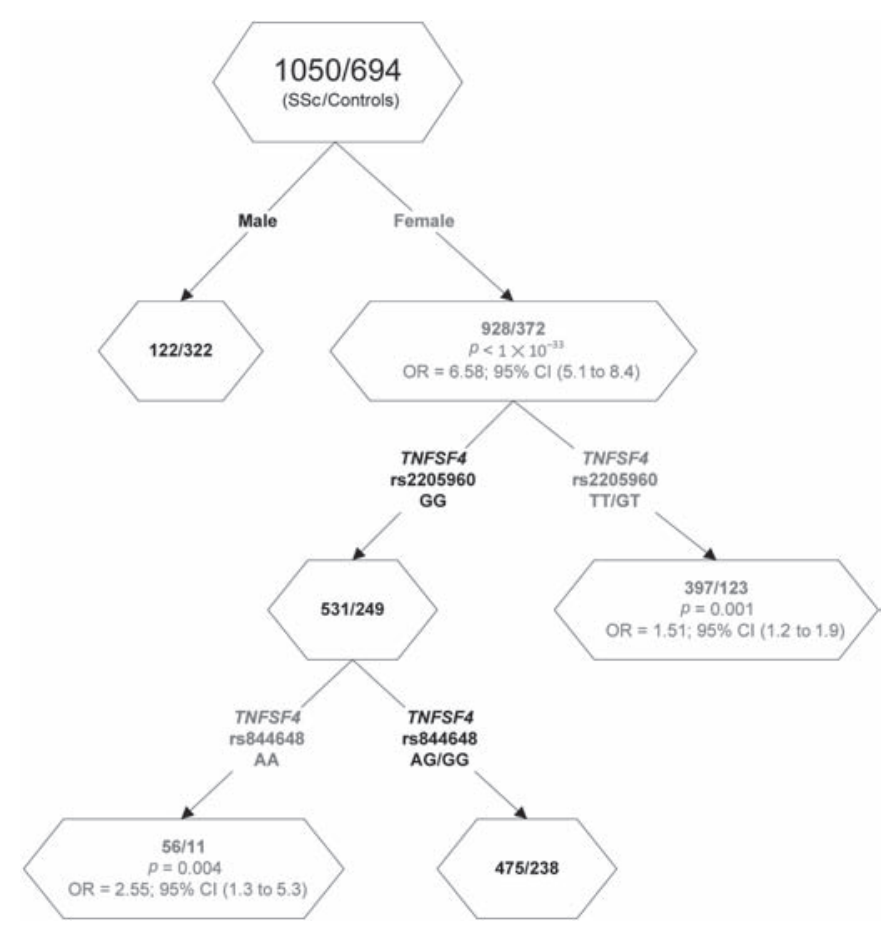

Figure 3 Cartesian and regression tree analysis (CART) showing an interaction between the tumour necrosis factor ligand superfamily member 4 gene (TNFSF4) single nucleotide polymorphisms (SNPs) rs2205960 and rs944648 in systemic sclerosis (SSc). Red test result denotes SSc susceptibility factors.

genotype $(\mathrm{OR}=1.51,95 \% \mathrm{CI} 1.2$ to 1.9$)$ consistent with a dominant model. The individuals with wild-type genotype 'GG' at rs2205960 were subsequently further split by the TNFSF4 variant (rs855648) into two subgroups: the 'AA' genotype of the TNFSF 4 variant increased risk for SSc as compared to the 'AG/ GG' genotypes (OR=2.55, 95\% CI 1.3 to 5.3). Therefore, this analysis uncovered a potential interaction between the rs 2205960 and rs855648 SNPs in the TNFSF4 gene region among women patients with SSc. This interaction between the two TNFSF 4 variants, rs2205960 and rs855648, was further tested and confirmed by an interaction model in logistic regression analysis $(\mathrm{p}=0.0004)$.

\section{DISCUSSION}

The current report is the first demonstration of an association of polymorphisms in the TNFSF4 gene region with susceptibility to SSc in North American Caucasians. These associations were similar when comparing $1 \mathrm{SSc}$ and $\mathrm{dSSc}$ with controls. We also identified particular SNPs in the TNFSF 4 gene region that are associated with the SSc-associated autoantibody subsets of SSc. Lastly, the exploratory method of analysis, CART, demonstrated a potential interaction between the TNFSF4 SNPs rs2205960 and rs855648 among Caucasian women patients with SSc that was confirmed by logistic regression analysis.

TNFSF4 encodes for the protein OX40 ligand (OX40L). OX40L is expressed on dendritic cells, macrophages, B cells, $\mathrm{T}$ cells, natural killer (NK) cells as well as non-immune cells such as endothelial cells and smooth muscle cells. ${ }^{38}$ It is the ligand for the OX40 which is expressed on CD4+ and CD8+ $\mathrm{T}$ cells, where it provides a costimulatory signal resulting in T cell proliferation, survival and cytokine production. ${ }^{38}$ Several studies have suggested that OX40-OX40L interaction may preferentially promote $\mathrm{T}$ helper (Th) 2 cytokines and may be a negative regulatory signal for interleukin (IL)17 production. ${ }^{39-41}$ More recently it has been suggested that OX40-OX40L interactions inhibit the formation of Foxp3+ regulatory $\mathrm{T}$ cells. ${ }^{42}$ In addition to $\mathrm{T}$ cell effects, OX40-OX40L interactions also have been reported to be critical in differentiation of $B$ cells and antibody production. ${ }^{43-45}$ Lastly, it has been reported that plasmacytoid dendritic cells may regulate $\mathrm{T}$ helper responses through a balance of OX40L expression and type I interferon expression such that OX40L alone induces a Th2 response and OX40L with type I interferon may have a Th1 inducing effect. ${ }^{46}$ Together these studies suggest an important role for OX40-OX40L interactions in critical immunoregulatory checkpoints that are likely involved in the development of autoimmunity. Accordingly, OX40L transgenic mice on the C57/BL6 background develop organ specific inflammation and targeted strategies to block OX40-OX40L interactions have prevented the development of diabetes in the non-obese diabetic mouse and experimental autoimmune encephalomyelitis, a model for multiple sclerosis. ${ }^{47-49}$ It is likely that these pathways and OX40L-dependent immune alterations are important in the development of SSc. Indeed serum levels of OX40 have been reported to be elevated in patients with $\mathrm{SSc}$, suggesting that the OX40-OX40L pathway may be involved in SSc. ${ }^{50}$ However, specifically how the OX40L polymorphisms lead to the development of SSc is unknown.

The current report adds TNFSF 4 to the list of genes that are associated with SSc susceptibility which includes major histocompatibility complex (MHC) class II, PTPN22, AIF1, IRF5, STAT4 and FAS. ${ }^{8} 21-233651$ Furthermore, given the association of TNFSF 4 with SLE as well as SSc, it is likely that TNFSF4 is a gene that confers risk to multiple autoimmune diseases. Why individual patients will develop a specific autoimmune disease such as SLE, RA, or SSc remains unknown. Functional studies of the polymorphisms in these genes as well as studies of genegene and gene-environment interactions are necessary to better understand how the genetic associations lead to specific autoimmune diseases.

Our study, despite its large size, is limited to the North American Caucasian population. Current efforts are underway to confirm these findings in other populations. However, we believe that the prior association of TNFSF 4 with SLE and the emerging concepts of shared autoimmune genes provide some validation of our novel findings in SSc. Furthermore, the odds ratios that we observed in the current study are small, but statistically significant. It is well established that SSc and other autoimmune diseases are complex polygenic diseases and it will be important to investigate for interactions among these candidate genes in the future. Another important limitation to consider when interpreting the findings of the current study is that our data demonstrate an association of a region $5^{\prime}$ of the TNFSF4 gene region with SSc susceptibility. These SNPs have been reported to be within the promoter region of TNFSF 4 but their specific regulatory role is not known. ${ }^{11}$ Higher resolution genotyping or DNA sequencing of this region gene will help to identify the SNPs involved in SSc pathogenesis and also determine the variation in genetic structure of this gene in various populations.

To summarise, the current report identifies TNFSF4 as a susceptibility gene for the development of SSc. These data further our understanding of the complex immunopathogenesis of SSc and provide additional support for the emerging concept of shared genes in multiple autoimmune diseases. 
Acknowledgements We would like to thank our patients and healthy volunteers who participated in these cohorts, without whom these studies would not be possible.

Ethics approval This study was conducted with the approval of the University of Texas Health Science Center at Houston Medical School IRB (Committee for the Protection of Human Subjects).

Contributors All authors contributed to the following criteria for the manuscript authorship conception and design, or analysis and interpretation of data drafting the article or revising it critically for important intellectual content and final approval of the version to be published.

\section{Provenance and peer review Not commissioned; externally peer reviewed.}

\section{REFERENCES}

1. Plenge RM, Padyukov $L$, Remmers $E F$, et al. Replication of putative candidate-gene associations with rheumatoid arthritis in $>4,000$ samples from North America and Sweden: association of susceptibility with PTPN22, CTLA4, and PADI4. Am J Hum Genet 2005;77:1044-60.

2. Concannon P, Rich SS, Nepom GT. Genetics of type 1 A diabetes. N Engl J Med 2009;360:1646-54

3. Harley IT, Kaufman KM, Langefeld CD, et al. Genetic susceptibility to SLE: new insights from fine mapping and genome-wide association studies. Nat Rev Genet 2009;10:285-90.

4. Begovich AB, Carlton VE, Honigberg LA, et al. A missense single-nucleotide polymorphism in a gene encoding a protein tyrosine phosphatase (PTPN22) is associated with rheumatoid arthritis. Am J Hum Genet 2004;75:330-7.

5. Onengut-Gumuscu S, Ewens KG, Spielman RS, et al. A functional polymorphism $(1858 \mathrm{C} / \mathrm{T})$ in the PTPN22 gene is linked and associated with type I diabetes in multiplex families. Genes Immun 2004;5:678-80.

6. Orozco G, Sánchez E, González-Gay MA, et al. Association of a functional singlenucleotide polymorphism of PTPN22, encoding lymphoid protein phosphatase, with rheumatoid arthritis and systemic lupus erythematosus. Arthritis Rheum 2005;52:219-24.

7. Remmers EF, Plenge RM, Lee AT, et al. STAT4 and the risk of rheumatoid arthritis and systemic lupus erythematosus. N Engl J Med 2007;357:977-86.

8. Rueda B, Broen J, Simeon C, et al. The STAT4 gene influences the genetic predisposition to systemic sclerosis phenotype. Hum Mol Genet 2009;18:2071-7.

9. Cunninghame Graham DS, Graham RR, Manku H, et al. Polymorphism at the TNF superfamily gene TNFSF4 confers susceptibility to systemic lupus erythematosus. Nat Genet 2008;40:83-9.

10. Chang YK, Yang W, Zhao M, et al. Association of BANK1 and TNFSF4 with systemic lupus erythematosus in Hong Kong Chinese. Genes Immun 2009;10:414-20.

11. Mälarstig A, Eriksson $P$, Rose $L$, et al. Genetic variants of tumor necrosis factor superfamily, member 4 (TNFSF4), and risk of incident atherothrombosis and venous thromboembolism. Clin Chem 2008;54:833-40.

12. Mälarstig A, Eriksson $P$, Rose L, et al. Genetic variants of tumor necrosis factor superfamily, member 4 (TNFSF4), and risk of incident atherothrombosis and venous thromboembolism. Clin Chem 2008;54:833-40.

13. Olofsson PS, Söderström LA, Jern C, et al. Genetic variants of TNFSF4 and risk for carotid artery disease and stroke. J Mol Med 2009;87:337-46.

14. Wang X, Ria M, Kelmenson PM, et al. Positional identification of TNFSF4, encoding OX40 ligand, as a gene that influences atherosclerosis susceptibility. Nat Genet 2005;37:365-72.

15. Charles C, Clements P, Furst DE. Systemic sclerosis: hypothesis-driven treatment strategies. Lancet 2006;367:1683-91.

16. Reveille JD, Fischbach M, McNearney T, et al.; GENISOS Study Group. Systemic sclerosis in 3 US ethnic groups: a comparison of clinical, sociodemographic, serologic, and immunogenetic determinants. Semin Arthritis Rheum 2001; 30:332-46

17. Baechler EC, Batliwalla FM, Karypis G, et al. Interferon-inducible gene expression signature in peripheral blood cells of patients with severe lupus. Proc Natl Acad Sci USA 2003;100:2610-15.

18. Hua J, Kirou K, Lee $\mathbf{C}$, et al. Functional assay of type I interferon in systemic lupus erythematosus plasma and association with anti-RNA binding protein autoantibodies. Arthritis Rheum 2006;54:1906-16.

19. Tan FK, Zhou X, Mayes MD, et al. Signatures of differentially regulated interferon gene expression and vasculotrophism in the peripheral blood cells of systemic sclerosis patients. Rheumatology (Oxford) 2006;45:694-702.

20. Graham RR, Kyogoku C, Sigurdsson S, et al. Three functional variants of IFN regulatory factor 5 (IRF5) define risk and protective haplotypes for human lupus. Proc Natl Acad Sci USA 2007;104:6758-63.

21. Dieudé $\mathbf{P}$, Guedj M, Wipff J, et al. Association between the IRF5 rs2004640 functional polymorphism and systemic sclerosis: a new perspective for pulmonary fibrosis. Arthritis Rheum 2009;60:225-33.

22. Gourh P, Tan FK, Assassi S, et al. Association of the PTPN22 R620W polymorphism with anti-topoisomerase I- and anti-centromere antibody-positive systemic sclerosis. Arthritis Rheum 2006;54:3945-53.
23. Liakouli V, Manetti M, Pacini A, et al. The $-670 \mathrm{G}>$ A polymorphism in the FAS gene promoter region influences the susceptibility to systemic sclerosis. Ann Rheum Dis 2009:68:584-90.

24. Kanemitsu S, Ihara K, Saifddin A, et al. A functional polymorphism in fas (CD95/ APO-1) gene promoter associated with systemic lupus erythematosus. J Rheumatol 2002;29:1183-8

25. Lee YH, Kim YR, Ji JD, et al. Fas promoter -670 polymorphism is associated with development of anti-RNP antibodies in systemic lupus erythematosus. J Rheumatol 2001;28:2008-11.

26. Mayes MD. The establishment and utility of a population-based registry to understand the epidemiology of systemic sclerosis. Curr Rheumatol Rep 2000;2:512-16.

27. Arnett FC, Reveille JD, Goldstein R, et al. Autoantibodies to fibrillarin in systemic sclerosis (scleroderma). An immunogenetic, serologic, and clinical analysis. Arthritis Rheum 1996;39:1151-60.

28. Subcommittee for scleroderma criteria of the American Rheumatism Association Diagnostic and Therapeutic Criteria Committee. Preliminary criteria for the classification of systemic sclerosis (scleroderma). Arthritis Rheum 1980; 23:581-90.

29. Hummers LK, Hall A, Wigley FM, et al. Abnormalities in the regulators of angiogenesis in patients with scleroderma. J Rheumatol 2009;36:576-82.

30. Assassi S, Arnett FC, Reveille JD, et al. Clinical, immunologic, and genetic features of familial systemic sclerosis. Arthritis Rheum 2007;56:2031-7.

31. LeRoy EC, Black C, Fleischmajer R, et al. Scleroderma (systemic sclerosis): classification, subsets and pathogenesis. J Rheumatol 1988;15:202-5.

32. Fritzler MJ, Kinsella TD. The CREST syndrome: a distinct serologic entity with anticentromere antibodies. Am J Med 1980;69:520-6.

33. Benjamini Y, Hochberg Y. Controlling the false discovery rate: a practical and powerful approach to multiple testing. Journal of the Royal Statistical Society 1995; 57:289-300.

34. Zhang $\mathbf{H}$, Bonney $\mathbf{G}$. Use of classification trees for association studies. Genet Epidemiol 2000;19:323-32.

35. Meyer OC, Fertig N, Lucas M, et al. Disease subsets, anti-nuclear antibody profile, and clinical features in 127 French and 247 US adult patients with systemic sclerosis. J Rheumatol 2007;34:104-9.

36. Alkassab F, Gourh P, Tan FK, et al. An allograft inflammatory factor 1 (AIF1) single nucleotide polymorphism (SNP) is associated with anti-centromere antibody positive systemic sclerosis. Rheumatology (Oxford) 2007;46:1248-51.

37. Wu X, Gu J, Grossman HB, et al. Bladder cancer predisposition: a multigenic approach to DNA-repair and cell-cycle-control genes. Am J Hum Genet 2006; 78:464-79.

38. Croft M. The role of TNF superfamily members in T-cell function and diseases. Nat Rev Immunol 2009;9:271-85.

39. Jenkins SJ, Perona-Wright G, Worsley AG, et al. Dendritic cell expression of OX40 ligand acts as a costimulatory, not polarising, signal for optimal Th-2 priming and memory induction in vivo. J Immunol 2007;179:3515-23.

40. Hoshino A, Tanaka Y, Akiba H, et al. Critical role for OX40 ligand in the development of pathogenic Th-2 cells in a murine model of asthma. Eur J Immunol 2003; 33:861-9

41. Li J, Li L, Shang X, et al. Negative regulation of IL-17 production by 0X40/0X40L interaction. Cell Immunol 2008;253:31-7.

42. Xiao X, Kroemer A, Gao W, et al. OX40/0X40L costimulation affects induction of Foxp3 + regulatory T-cells in part by expanding memory T-cells in vivo. J Immunol 2008;181:3193-201

43. Kato H, Kojima H, Ishii N, et al. Essential role of OX40L on B cells in persistent alloantibody production following repeated alloimmunisations. J Clin Immunol 2004:24:237-48

44. Stüber E, Neurath M, Calderhead D, et al. Cross-linking of OX40 ligand, a member of the TNF/NGF cytokine family, induces proliferation and differentiation in murine splenic B cells. Immunity 1995;2:507-21.

45. Stüber E, Strober W. The T cell-B cell interaction via OX40-OX40L is necessary for the T cell-dependent humoral immune response. J Exp Med 1996;183:979-89.

46. Ito $\mathbf{T}$, Amakawa $\mathrm{R}$, Inaba $\mathrm{M}$, et al. Plasmacytoid dendritic cells regulate Th cell responses through OX40 ligand and type I IFNs. J Immunol 2004;172:4253-9.

47. Murata K, Nose M, Ndhlovu LC, et al. Constitutive OX40/0X40 ligand interaction induces autoimmune-like diseases. J Immunol 2002;169:4628-36.

48. Pakala SV, Bansal-Pakala P, Halteman BS, et al. Prevention of diabetes in NOD mice at a late stage by targeting 0X40/0X40 ligand interactions. Eur J Immunol 2004;34:3039-46.

49. Nohara C, Akiba H, Nakajima A, et al. Amelioration of experimental autoimmune encephalomyelitis with anti-OX40 ligand monoclonal antibody: a critical role for OX40 ligand in migration, but not development, of pathogenic T cells. J Immunol 2001;:166:2108-15.

50. Komura K, Yoshizaki A, Kodera M, et al. Increased serum soluble OX40 in patients with systemic sclerosis. J Rheumato/ 2008;35:2359-62.

51. Reveille JD, Durban E, MacLeod-St Clair MJ, et al. Association of amino acid sequences in the HLA-DOB1 first domain with anti-topoisomerase I autoantibody response in scleroderma (progressive systemic sclerosis). J Clin Invest 1992:90:973-80. 


\section{Corrections}

Pravitt Gourh, Frank C Arnett, Filemon K Tan, et al. Association of TNFSF4 (OX40L) polymorphisms with susceptibility to systemic sclerosis. Ann Rheum Dis 2010;69:550-55. doi:10.1136/ ard.2009.116434. The article has been published without the funding source. The funding source is: CDMRP grant PRO64251. (Note: CDRMP stands for Congressionally Directed Medical Research Programs).

Ann Rheum Dis 2011;70:880. doi:10.1136/ard.2009.116434corr1 